Artigo de Revisão

\title{
Midazolan nasal no tratamento de crises convulsivas
}

\author{
Sueli Rizzutti ${ }^{1}$, Lucila Bizari Fernandes do Prado², Gilmar Fernandes do Prado³.
}

\begin{abstract}
RESUMO
Pacientes com crises agudas incluindo estado de mal epiléptico, podem apresentar dificuldades no tratamento que geralmente envolve um benzodiazepínico. As drogas mais amplamente utilizadas em pacientes com crises agudas incluem diazepam, lorazepam e midazolam. Nós revisamos a eficácia e a segurança das diversas formas de administração do midazolam. A administração intranasal e bucal do midazolam pode ser uma alternativa a administração retal e intravenosa do diazepam.
\end{abstract}

Unitermos: Administração intranasal; Administração bucal; Crises agudas; Midazolam.

\section{SUMMARY}

Patients with acute seizure, including status epilepticus, can prove difficult to treat and first line treatment generally involves a benzodiazepine drug. The most widely used drugs in the treatment of patients with acute seizures are diazepam, lorazepam and midazolam. We review the efficacy and safety of the ways of midazolam administration. Intranasal and buccal midazolam could be an altemative to rectal and intravenous diazepam.

Keywords: Intranasal Administration; Buccal administration; Acute seizures; Midazolam

\section{INTRODUÇÃO.}

No tratamento agudo de crises epilépticas o diazepam (DZP) é o benzodiazepínico (BZD) mais amplamente usado em crianças e adultos, Henry Gastaut (1965) foi o primeiro a utilizar o diazepam porvia endovenosa para o controle do estado de mal epiléptico. Porém o diazepam (DZP) tem algumas desvantagens, incluindo a tendência ao efeito cumulativo com doses repetidas, duração de ação muito curta, deve ser dado por via endovenosa ou retal e apresenta lenta absorção se dada por via intramuscular. Em situações agudas, os benzodiazepínicos podem ser dados por via retal, intramuscular e endovenosa. A administração endovenosa nem sempre é bem sucedida em crianças pequenas, principalmente em situações de emergência, a administração retal pode ser difícil em algumas situações como também a absorção retal pode ser muito variável. Os benzodiazepínicos são usados rotineiramente por via retal no tratamento de crises epilépticas

\footnotetext{
Traba lho realiza do: Disciplina de Neurologia - UNIFESP. São Paulo. Brasil.

1 - Neuropediatra

2 - Pediatra.

3 - Neurologista.
}

Endereço para Correspondência: Dra. Sueli Rizzutti - Rua Dorali, 72 - Guarulhos - SP - Telefone: 6461.3982 - e-mail: surizzutti@ig.com.br 
agudas, porém os cuidadores, monitores e professores são relutantes em utilizar esse meio de administração nas escolas, e também, as crianças mais velhas acham esse procedimento muito embaraçoso. Alguns autores tem explorado a administração intranasal e oral do midazolam ${ }^{1-3}$ - Assim, nós revisamos a eficácia e a segurança da administração oral e nasal do midazolam utilizado em alguns países como uma forma alternativa ao uso retal do diazepam.

\section{Diazepam e Lorazepam.}

O estado de mal epiléptico (EME) é a emergência neurológica mais freqüente, sendo necessário tratamento eficaz, imediato para 0 controle da atividade epiléptica, no sentido de que sejam prevenidas lesões neuronais e/ou outros distúrbios sistêmicos associados ${ }^{4}$. Quanto mais se atrasa o começo do tratamento, mais resistente ele se torna e o tratamento precoce pode reduzir a morbidade e a mortalidade. 0 trajeto de casa ao hospital pode atrasar o início do tratamento e reduzir a sua eficácia e o tratamento precoce pode reduzir o tempo de tratamento. Por isso é importante que haja medicamentos capazes de acabar com a crise rapidamente e que sejam fáceis de administrar.

Em nosso meio, a droga de escolha para o tratamento inicial do estado de mal epiléptico é o diazepam. Este apresenta meia-vida curta por redistribuição (menorque 1 hora) e grande volume de distribuição 1 a 2 l/Kg e por isso, embora seja uma droga com penetração imediata no SNC, a rápida disseminação para outros tecidos leva à queda acentuada no nível parenquimatoso cerebral, acarretando duração do efeito anticonvulsivante de apenas 15 a 30 minutos $^{5,6}$. Portanto, é fundamental a associação de uma segunda droga, com vida média mais longa, após o controle inicial das crises. A eficácia do DZP no controle das crises é de aproximadamente 85 a $90 \%{ }^{6,7}$. A via de utilização preferencial é a endovenosa, a diluição não é necessária, não se deve utilizar a via intramuscular, pois a absorção é muito lenta inclusive quando comparada com a via oral. O uso retal leva a uma absorção irregular, a medicação pode ser expulsa e também pode produzir uma sonolência prolongada pós ictal. 0 efeito colateral principal do DZP consiste na depressão sensorial e principalmente respiratória.

Nos últimos anos, outro BZD, o lorazepam (LZP) vem sendo preferido em vários centros, como a droga ideal para a abordagem inicial do EME. A via utilizada é a endovenosa, na dose de 0,05 a $0,1 \mathrm{mg} / \mathrm{kg} /$ dose (máximo $4 \mathrm{mg}$ ), em infusão de um a dois minutos ${ }^{8,9}$. A eficácia é de 80 a $100 \%$ e os efeitos colaterais são semelhantes ao DZP. A maior vantagem consiste na duração (12 a 24 horas), podendo ser utilizado como droga antiepiléptica única. Até o momento o LZP endovenoso não é comercializado no Brasil.

\section{Via de administração.}

A administração retal de medicações pode ter conotações pessoais e sociais tão importantes a ponto de não ser utilizada pelo próprio paciente principalmente adultos e adolescentes ${ }^{10}$. Os pais, cuidadores, monitores de acampamentos geralmente recusam essa via de administração sem uma supervisão sanitária, além de que o uso dessa via pode ser difícil durante uma crise epiléptica generalizada em um paciente adulto ou adolescente. Em outros casos se teme a acusação de abuso sexual em lugares públicos como escolas e acampamentos ${ }^{11,12}$. A administração oral e nasal pode serum avanço muito importante para o tratamento de crises epilépticas agudas, visto ser muito mais fácil a aceitação pessoal e social, levando a um tratamento mais precoce de crises epilépticas agudas.

Com isso, faremos uma revisão da literatura na utilização dos BZD por via intranasal e oral. O DZP é pouco solúvel em água e necessita da utilização de propilenoglicol para solubilizar a solução endovenosa ou de cremofor para a administração retal, nenhum destes solventes é adequado para o uso oral ou intranasal. Por outro lado o midazolam é solúvel em água facilitando seu uso oral e intra nasal. Faremos uma abordagem da farmacodinâmica do midazolam como também da sua eficácia e segurança para o uso intranasal e oral (Tabela 1). 


\section{MIDAZOLAN.}

O midazolam (MDZ) pertence a uma nova classe de benzodiazepínicos chamado imidazobenzodiazepínico (Figura 1) hidrossolúvel introduzido na prática clínica em 1982, com características farmacocinéticas e farmacodinâmicas bem conhecidas. Esta classe também inclui um receptor antagonista flumazenil. É indicado na prática clínica em adultos e crianças para sedação consciente antes e durante procedimentos diagnósticos ou terapêuticos com ou sem anestesia local, como pré medicação antes da indução anestésica, sedação em unidades de cuidados intensivos como também no tratamento de estados de mal epiléptico. 0 MDZ difere de outros agentes por apresentar efeitos clínicos rápidos e com curta duração de ação.

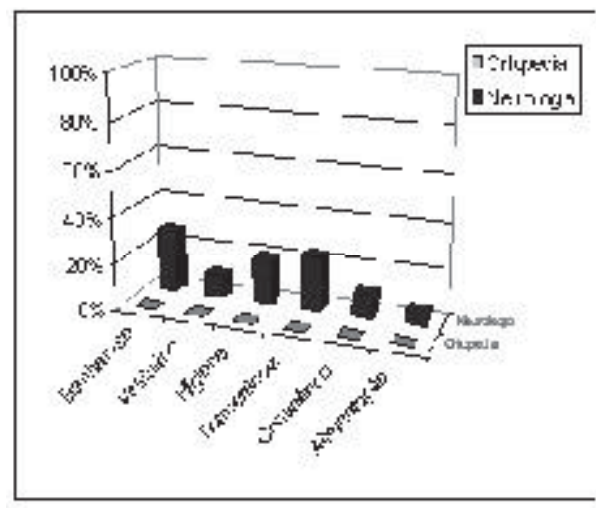

Figura 1 - Midazolan

O MDZ é um imidazobenzodiadiazepínico que é básico e solúvel em água no $\mathrm{pH}$ fisiológico. A base livre é pouco solúvel em água, porém no meio ácido da ampola que contém cloreto sódico, ácido clorídrico, hidróxido de sódio e água em um pH de 3,3, seu anel BZP se abre e permite que o MDZ seja solúvel em água de forma estável. Uma vez administrado, 0 anel BZP se fecha no $\mathrm{pH}$ fisiológico e o MDZ se torna lipossolúvel permitindo uma rápida penetração no cérebro.

\section{Absorção.}

O MDZ após a administração oral é rapidamente absorvido pelo trato gastrointestinal. A sonolência tem sido observada após 15 minutos da dose oral, com pico de efeito após 30 a 90 minutos $^{13}$. A duração de ação após a administração intranasal é semelhante a oral, entretanto o início da sedação é mais rápido ${ }^{14,15}$. A administração retal é segura e efetiva com pico de ação em 8 a 29 minutos após a administração ${ }^{15}$. Ao contrário do diazepam e clordiazepóxido, os quais tem uma absorção intramuscular errática e incompleta, o MDZ é rapidamente absorvido após a injeção intramuscular, com uma biodisponibilidade de mais de 90\%, o início da sedação ocorre após 5 minutos da injeção com pico em 15 a 30 minutos $^{16}$. 0 início da sedação pode ser visto em 3 minutos após a infusão endovenosa ${ }^{13}$.

\section{Distribuição.}

MDZ tem um volume de distribuição de 1,0$2,5 \mathrm{l} / \mathrm{kg}$ em indivíduos saudáveis. É rapidamente distribuído após a administração endovenosa. MDZ é altamente ligado a proteínas plasmáticas, primariamente a albumina, com uma fração livre de 4\%. A meia-vida de distribuição de apenas 15 minutos do MDZ mostra sua solubilidade relativamente alta em $\mathrm{pH}$ fisiológico.

\section{Metabolização.}

O MDZ, como outros BZP, é biotransformado no sistema microssomal hepático através da ahidroxilação e glicuronização. Inicialmente o MDZ é hidroxilado pelo sistema citocromo P450-3A4 para a-hidroxi-midazolam e uma pequena quantidade em metabólitos inativos. Estes metabólitos são então eliminados na urina como conjugados glicuronizados. O a-hidroximidazolam é farmacologicamente ativo e tem propriedades sedativas equivalente ao MDZ. Inibidores do sistema citocromo P450 como a cimetidina, fluconazol, eritromicina, ranitidina, claritromicina podem reduzir o seu metabolismo 
diminuindo sua eliminação. A depressão do SNC pode ser potencializada por outros depressores como os opióides, antipsicóticos, fenobarbital, antihistamínicos e álcool.

\section{Eliminação.}

O MDZ tem uma meia-vida de eliminação de 1,5 a 3 horas comparado com a do diazepam de mais de 20 horas. Entretanto, a duração da ação farmacológica é de 60 a 120 minutos ${ }^{17}$. 0 metabólito a-hidroxi-midazolam tem uma meiavida de eliminação de 1 hora em humanos. Cerca de $90 \%$ da administração oral do MDZ é excretada em 24 horas. A maior eliminação é realizada pelo rim e uma menor proporção (menos de 10\%) pelas fezes em 5 dias. A meiavida se prolonga a 6-12 horas em recémnascidos, mais curta de 1 a 1,5 horas em crianças de 3 a 10 anos, 0,5 a 2 horas na gravidez, em 10 horas em pacientes com mais de 60 anos e 5,9 horas em obesos.

\section{Farmacologia.}

O MDZ tem uma alta afinidade por receptores benzodiazepínicos no sistema nervoso central. Os receptores benzodiazepínicos tem sido identificados em vários tecidos incluindo coração e musculatura esquelética, porém o maior predomínio ocorre no sistema nervoso central. A ação dos BZDs é no sítio receptor para o maior neurotransmissor inibitório do sistema nervoso central, o ácido gama-aminobutírico (GABA). Liberado dos neurônios GABAérgicos, o GABA se liga aos receptores $G_{A B A_{A}}$ e $G A B A$ B. $O s$ benzodiazepínicos aumentam a inibição mediada pelo $\mathrm{GABA}_{\mathrm{A}}$, mas não tem ação na inibição mediada pelo receptor $G A B A_{B}$. é uma proteína macromolecular que forma um canal complexo, íon seletivo cloreto e contém sítios ligantes para GABA, barbitúricos, anestésicos, $B Z D$, esteróides. Este sítio é parte do sítio receptor $G_{A B A_{A}}$ e existe em uma variedade de númeroe formas (BZDI, BZDII,
BZDIII). Os BZD potencializam a ação inibitória do GABA pelo aumento do fluxo dos íon cloreto através dos canais iônicos. 0 aumento do fluxo dos íons cloretos para dentro da célula diminui a habilidade da célula iniciar o potencial de ação. Os BZD aumentam a freqüência de abertura do canal e bloqueiam descargas mantidas de alta freqüência, o mecanismo deste último tipo de ação é incerto e é visto em elevadas concentrações terapêuticas. O MDZ é somente aprovado pelo FDA para a administração endovenosa e intramuscular, entretanto a via retal e intranasal são empregadas freqüentemente ${ }^{18,19}$.

\section{Efeitos sedativos}

O MDZ tem propriedades ansiolíticas, antiepilépticas, sedativas, hipnóticas, amnésticas e também pode atuar como relaxante muscular. É utilizado em urgência para aliviar a ansiedade e conseguir analgesia e amnésia quando se utiliza procedimentos cirúrgicos e ortopédicos em pacientes conscientes. A sedação é conseguida com concentração de $30-100 \mathrm{ng} / \mathrm{ml}$, por via intramuscular ou endovenosa. Ramoska et al. $(1991)^{20}$ demonstraram adequada sedação com a dose de 1 a 3mg de MDZ. A dose recomendada inicialmente endovenosa é de $1 \mathrm{mg}$ ( não mais do que $0,03 \mathrm{mg} / \mathrm{kg}$ ) lentamente. Uma dose de 0,02 a $0,03 \mathrm{mg} / \mathrm{kg}$ pode ser repetida com monitorização adequada do nível de sedação. $A$ dose intramuscular é de 0,07 a 0,08mg/kg. Em pacientes crônicos e em pacientes mais velhos a dose deve ser dividida pela metade. Em estudos comparativos, o efeito sedativo do MDZ endovenoso para cirurgia maxilofacial foi semelhante ao diazepam endovenoso, porém com menos dor no local da injeção e com mais amnésia ${ }^{21} .0 \mathrm{MDZ}$ por via intramuscular foi mais eficaz que o haloperidol em pacientes com agitação aguda22. Pode ser usado por via retal e oral para evitar a ansiedade causada pelas vias parenterais, principalmente em crianças. Estudos comparativos indicam que o MDZ retal tem um efeito mais rápido e produz mais amnésia e menos depressão respiratória do que o diazepam 
retal em pacientes que se submeteram a endoscopia ${ }^{23}$.

\section{Uso do midazolam intranasal e oral.}

As vias intranasal e oral (Tabela 2) são vias de fácil acesso que podem ser utilizadas na urgência. A administração de 0,1 e 0,2mg/kg de MDZ intranasal alcança concentrações de 40 a $70 \mathrm{ng} / \mathrm{ml} \mathrm{em} 1$ a 3 minutos da administração, que são suficientes para produzir a sedação em adultos. O MDZ tem um gosto amargo, mas pode ser diluído no suco. 0 pico de $104 \mathrm{ng} / \mathrm{ml}$ se alcança após 12 minutos da administração de $0,2 \mathrm{mg} / \mathrm{kg}$, com uma biodisponibilidade de $55 \%$. A mucosa nasal é altamente vascularizada levando a uma maior absorção, sendo que pode chegar a uma biodisponibilidade de até $83 \%$ muito maior que a biod is ponibilidade de $44-66 \%$ da via oral. Temos que levar em consideração que se deglute líquido quando se administra pela via intranasal caindo a biodisponibilidade para 50-57\%. Para a administração intranasal se utiliza $\mathrm{da}$ solução parenteral de $5 \mathrm{mg} / \mathrm{ml}$. 0 procedimento mais habitual é conectar um cateter de plástico em uma seringa e gotejar o volume calculado por peso, instilando metade da dose em cada narina. Recentemente tem se sugerido o uso de aerossóis e o uso de solubilizantes específicos como a ciclodextrina para a administração intranasal. A farmacologia dos aerossóis se tem estudado em voluntários sãos $^{24}$ e em adultos que necessitam de sedação ${ }^{25}$. Os aerossóis podem aumentar a absorção na parte anterior e superior das fossas nasais, reduzir a quantidade que se deglute e passar direto ao sistema nervoso centra ${ }^{25}$.

0 uso de solubilizantes como a ciclodextrina permite aumentar a concentração da solução de 5 a $17 \mathrm{mg} / \mathrm{ml}$ e elevar o pH de 3,3 a 4,3. A maior concentração reduz o volume que se administra, diminuindo a deglutição e a mudança do pH, reduz a irritação local e acelera a absorção. A absorção do MDZ intranasal não ocorre em um $\mathrm{pH}$ de 3,5 e sim de 5,5 a 7,526. 0 pH da solução de ciclodextrina leva a uma absorção imediata.
O MDZ pode também ser usado por via sublingual e bucal. Tem-se sugerido que 0 acesso ao cérebro através da mucosa olfatória pode ser mais rápido quando MDZ é administrado por via intranasal, porém não se tem encontrado diferenças importantes da rapidez em produzir o efeito sedativo entre as vias de administração bucal e nasal27. Connors e Terndrup (1994) ${ }^{28}$ compararam a eficácia da administração intranasal e a oral em crianças para a reparação de lacerações e não observaram diferenças na eficácia, entretanto a administração intranasal foi associada com maior desconforto. A administração retal de $0,3 \mathrm{mg} / \mathrm{kg}$ diluída em $5 \mathrm{ml}$ de solução salina é uma alternativa de administração.

\section{Toxicidade.}

O MDZ foi aprovado para o seu uso em 1986, nos Estados Unidos e após 6 meses de sua liberação, esta droga foi associada com 13 fatalidades relacionadas a depressão respiratória, parada cardíaca principalmente em pacientes mais velhos ${ }^{29}$. Há também relatos de parada respiratória em pacientes pediátricos ${ }^{30}$. Diante dos fatos ocorridos a dose recomendada foi reduzida de 0,1-0,2 mg/kg para $0.03 \mathrm{mg} / \mathrm{kg}$. A maioria dos casos fatais ocorreram durante 0 uso de MDZ combinado com opióides tais como o fentanil. A associação de fentanil e MDZ foi associada com hipoventilação em mais do que $90 \%$ dos pacientes e apnéia em $50 \%{ }^{31}$. Outros efeitos adversos com MDZ são menos comuns. Arcos relatou 3 episódios de bigeminismo e trigeminismo e de taquicardia ventricular, as quais foram auto-limitadas e se resolveram em 2 a 4 horas da administração de $M D Z^{32}$. Yakel et al. $(1992)^{33}$ relataram um caso de angioedema e bronco-constrição após MDZ endovenoso. Esses dados sugerem a prática de monitorizar a sedação consciente com oxímetro e monitor cardíaco e o equipamento de ressuscitação cardiopulmonar deve estar sempre disponível. $\mathrm{Na}$ maioria dos casos de hipoventilação e apnéia, os pacientes respondem ao oxigênio $100 \%$ e 
estimulação verbal. Outros efeitos adversos observados incluem vômitos, tosse, náuseas e soluços. Em geral, os soluços desaparecem após alguns minutos e são a manifestação mais comum em adultos e crianças ${ }^{21,34}$.

Quando se administra o MDZ intranasal como sedativo, não se descreve efeitos cardiorespiratórios graves ao utilizar a dose de $0,2 \mathrm{mg} / \mathrm{kg}$. Tem-se descrito uma sensação de queimação, dor, irritação, irritação nasal, sensação desagradável na nasofaringe e sabor amargo, esses efeitos irritantes são atribuídas ao pH ácido da solução 27,28,35.

\section{E ficácia do Midazolam intranasale oral.}

A via intra nasal pode ter algumas dificuldades quando há movimentos intensos da cabeça e pode ser ineficaz quando há secreções nasais abundantes, considerando esses aspectos, a administração por via oral é uma outra possibilidade. A absorção através da mucosa bucal mediante a administração bucal (entre os dentes e a bochecha) ou sublingual é rápida; a mucosa bucal é ricamente vascularizada, a biodisponibilidade do MDZ pode chegar a ser $75 \%$ semelhante ao intranasal. Essa via pode facilitar a administração, pode ser dado um volume maior, tem menor risco de expulsar a medicação pela respiração, porém tem o risco de ser deglutido. Descreveremos a seguir alguns estudos após revisão sobre o uso de MDZ intranasal e oral.

0 primeiro estudo que mostra a utilização de medicações por via intranasal foi o de O'Regan et al. (1996) ${ }^{1} .0$ estudo compreendeu 19 crianças com a idade de 19 meses a 14 anos com epilepsia de difícil controle. O MDZ foi administrado lentamente gota a gota nas fossas nasais; em 15 crianças houve melhora eletrencefalográfica e cessação das crises, em 14 crianças houve o aparecimento de uma atividade beta induzida pela medicação. 0 tempo médio de aparecimento da atividade beta foi de 111,5 segundos. A redução dos spikes antes e depois do MDZ foi estatisticamente significante, a melhora da atividade elétrica cerebral de base foi também estatisticamente significante. As crianças e os pais consideraram o método aceitável.

Kendall et al. (1997) $)^{36}$ descrevem a eficácia do MDZ intranasal em dois pacientes com estado de mal epiléptico. Uma criança de 2 anos que mostrou controle das crises após a administração de 1,6 mg de MDZ intranasal por duas vezes, e a outra paciente de 53 anos que cessou o estado de mal após a administração de $10 \mathrm{mg}$ de MDZ intranasal.

Scott et al. (1997) ${ }^{37}$ descrevem 22 casos de crises prolongadas em 13 crianças com epilepsia refratária institucionalizadas em que utilizaram MDZ sublingual $(0,5 \mathrm{mg} / \mathrm{kg}$ no máximo $10 \mathrm{mg})$ com uma seringa sem agulha, demonstraram uma eficácia de $91 \%$, e não observaram alterações cardiores piratórias.

Scheepers etal. (1998) ${ }^{38}$ descrevem o uso de MDZ intranasal em dois adultos com déficit mental no controle de crises, o primeiro paciente (25 anos) pertencia a uma instituição e o MDZ substituiu o DZP por via retal com sucesso; a segunda paciente (20 anos) foi substituído o tratamento com BZD oral e retal pelo MDZ intranasal com boa eficácia. O MDZ intranasal foi administrado em uma seringa de $2,5 \mathrm{ml} \mathrm{com}$ cateter nasogástrico neonatal. Os autores preconizam a dose de $5 \mathrm{mg}$ para adultos com menos de $50 \mathrm{~kg}$ e a dose de $10 \mathrm{mg}$ para adultos com mais de $50 \mathrm{~kg}$. Os pais e cuidadores não tiveram problemas com a administração.

Lahat et al. (1998) ${ }^{39}$ mostram a eficácia do MDZ intranasal em um estudo aberto de 20 crianças com a idade de 6 meses a 16 anos, com controle das crises em 19 de 20 pacientes (95\%) em 2,5 a 5 minutos sem recidivas em 60 minutos.

J eannet et al. (1999) ${ }^{40}$ mostram a eficácia do MDZ em crianças de 1 dia a 16 anos (11 em casa e 17 em hospital de urgência). A dose preconizada foi de $0,2 \mathrm{mg} / \mathrm{kg}$, administrou-se a metade da dose em cada narina. Em dois casos 
a administração foi sublingual, nenhuma criança teve efeitos colaterais importantes, a tolerabilidade foi boa, os pais consideraram a administração cômoda e uma menor sonolência pós ictal quando comparado ao uso de DZP retal.

Chattopadhyay et al. (1999) ${ }^{12}$ descrevem uma análise retrospectiva da administração do MDZ bucal em 78 convulsões prolongadas tratadas ambulatorialmente (5 a $10 \mathrm{mg}$ ). O MDZ foi administrado em 2 a 60 minutos do começo da convulsão pelos pais e cuidadores e foi eficaz em $87 \%$, observou-se depressão respiratória em 7 de 78 episódios, e nenhuma reação adversa relevante.

Scott et al. (1999) ${ }^{41}$ realizaram o primeiro ensaio clínico comparando a eficácia e a segurança entre o MDZ bucal e DZP retal em 42 crianças com crises prolongadas institucionalizadas (5 a 19 anos). O MDZ foi utilizado na dose de $10 \mathrm{mg}$ em uma seringa com $2 \mathrm{ml}$ de solução ( $5 \mathrm{mg} / \mathrm{ml})$. Instilou-se o MDZ por toda a mucosa bucal. Se os dentes estavam fechados foi instilado entre os dentes e a bochecha. O DZP retal foi administrado na dose de $10 \mathrm{mg}$ mediante um preparad o comercial retal. A eficácia do MDZ foi de $75 \%$, e do DZP (59\%), essa diferença não foi significante como também o tempo para cessar as crises. Não se observaram efeitos cardiorespiratórios relevantes em nenhum dos grupos.

Finsgin et al. $(2000)^{42}$ administraram MDZ intranasal em 16 crianças na dose de $0,2 \mathrm{mg} / \mathrm{kg}$ e controlaram a freqüência cardíaca, respiratória, a pressão arterial e a saturação de $\mathrm{O}_{2}$ Em 13 crianças o MDZ foi efetivo, em 3 crianças outras medicações foram utilizadas, em uma criança houve diminuição da freqüência respiratória de 40 para $26 /$ minuto, em outra a freqüência cardíaca aumentou de 126 para 166/minuto, sem alteração da saturação do $\mathrm{O}_{2}$ e com recuperação em 5 minutos.

Scheepers et al. $(2000)^{10}$ administraram MDZ intranasal em 84 episódios de crises prolongadas, em 22 pacientes de 12 a 72 anos com epilepsia e institucionalizados. No início foi administrado com uma seringa conectada a um tubo nasogástrico neonatal e posteriormente se utilizou uma pipeta Pasteur. Em pacientes com muito movimento de cabeça se administrou via oral; a dose preconizada foi de $5 \mathrm{mg}$ para pacientes com menos de $50 \mathrm{~kg}$ e $10 \mathrm{mg}$ para pacientes com mais de $50 \mathrm{~kg}$. Não se observaram efeitos colaterais relevantes, nos pacientes em que se obteve boa resposta continuou a ser administrado pelos pais e cuidadores.

Lahat et al. $(2000)^{2}$ realizaram o primeiro ensaio comparativo do uso de MDZ intranasal $(1,2 \mathrm{mg} / \mathrm{kg})$ com o DZP endovenoso $(0,3 \mathrm{mg} / \mathrm{kg})$ no tratamento de crises febris prolongadas (mais de 10 minutos) em 47 crianças de 6 meses a 5 anos. O MDZ foi eficaz em 23 de 26 convulsões (88\%; 21 crianças); o DZP em 24 de 26 convulsões (92\%; 23 crianças): não houve diferença significativa. Nenhuma das crianças apresentou efeitos cardiorespiratórios nem outros efeitos secundários.

Fisgin et al. (2002) ${ }^{43}$ realizaram um ensaio clínico comparando a eficácia e a segurança do MDZ intranasal com o DZP retal no tratamento de convulsões agudas em 45 crianças de 1 mês a 13 anos na urgência. Em todas as crianças foi administrado 02, foi realizada a monitorização cardíaca, a saturação de 02, a freqüência respiratória e foi mantida uma via endovenosa de acesso. O MDZ foi eficaz em 20 dos 23 episódios (87\%) e o DZP retal em 13 de 22 pacientes $(59 \%)$, tendo sido significantemente maior a resposta ao MDZ intranasal. Não se observou nenhuma reação adversa relevante. Os dois últimos estudos não podem ser extrapolados para o meio extra hospitalar e nem em adultos (validade externa).

\section{CONCLUSÃO.}

O MDZ administrado intranasal e bucal parece tão eficaz e seguro quanto o DZP administrado intravenoso e retal 2,41,42. A recuperação pós crítica parece ser mais rápida 
e também, pode tratar episódios de agressividade e agitação pós-ictal, melhorando muito a qualidade de vida dos pacientes.

As vantagens mostradas pelos estudos sugerem a aplicação da medicação em lugares públicos pelos pais e cuidadores sem a conotação pessoal e social. Com a facilidade de administração pode-se reduzir o tempo do início do tratamento em episódios agudos e prolongados de crises epilépticas, melhorando a resposta e reduzindo a morbidade e mortalidade. Esta via de administração pode também facilitar a utilização da medicação por cuidadores em ambulatório, principalmente em pacientes adultos e adolescentes, e muitas vezes pode ser utilizado pelo próprio paciente como profilático quando tem uma aura.

Os estudos $2,41,42$ sugerem que o MDZ intranasal e bucal pode ser tão eficaz e seguro quanto o DZP retal e endovenoso para o tratamento precoce de crises agudas. Entretanto, não temos encontrado estudos controlados (ensaios clínicos bem desenhados) do uso de MDZ bucal em crianças pequenas e MDZ intranasal em adultos ${ }^{44-46}$, e também nenhum estudo controlado em ambulatório com a administração do MDZ por cuidadores, como também o seu uso em estado de mal epiléptico.

Lembramos que a solução parenteral de $5 \mathrm{mg} /$ $\mathrm{ml}$ para a administração intranasal produz irritação devido ao pH ácido, e o volume pode ser grande em adolescentes e adultos, podendo facilitar a deglutição e diminuir a eficácia. A melhora do meio de veiculação como o uso de aerossóis com soluções mais concentradas, pode minimizar os efeitos adversos locais.

Considerando a classificação dos níveis de evidência segundo Suzane Fletcher ${ }^{47}$ e David Sackett ${ }^{48}$, os resultados mostrados não devem ser extrapolados para o uso ambulatorial e por cuidadores sem a supervisão médica. São necessários ensaios clínicos mais amplos e com maior número de participantes para a sua utilização de uma forma mais científica e segura.

\begin{tabular}{|lc|c|c|c|}
\hline & 0 & 1 & 2 & 3 \\
Banhar-se & $48 \%$ & $5 \%$ & $19 \%$ & $28 \%$ \\
\hline Vestuário & $57 \%$ & $0 \%$ & $33 \%$ & $10 \%$ \\
\hline Higiene & $67 \%$ & $0 \%$ & $14 \%$ & $19 \%$ \\
\hline Transferência & $52 \%$ & $10 \%$ & $14 \%$ & $24 \%$ \\
Continência & $76 \%$ & $10 \%$ & $4 \%$ & $10 \%$ \\
\hline Alimentação & $95 \%$ & $0 \%$ & $0 \%$ & $5 \%$ \\
\hline
\end{tabular}

Tabela 1 - Farmacocinética

\begin{tabular}{|c|c|c|c|c|}
\hline & $\mathbf{u}$ & ר & $=$ & $\mathbf{y}$ \\
\hline Uanhar-F= & $U+3,30$ & 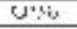 & $70 \%$ & 0,0 \\
\hline Vontusto & $\operatorname{sen} 2$ & 1130 & $a^{n}=$ & $\cos$ \\
\hline HIrilorente & $a=-i$, & $=-i$ is & $n=\%$ & $n=\ldots$ \\
\hline 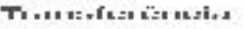 & $35 \%$ & ras & $n \approx$. & $a \cdots$ \\
\hline 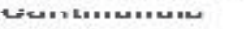 & 7135,46 & sese & $12 \div$ & $\ggg:$ \\
\hline Allmonxas:a0 & 7 urb. & $0:=$ & $w x^{2}$ & $u=$ \\
\hline
\end{tabular}




\section{REFERÊ N CIAS BIBLIOG RÁFICAS}

1-0'Regan ME, Brown J K, Clarke M. Nasal rather than rectal benzodiazepines in the management of acute childhood seizures? Dev Med Child Neurol 1996; 38: 1037-1045.

2-Lahat E, Goldman M, Barr J, Bistritzer, Berkovitch M. Comparison of intranasal midazolam with intravenous diazepam for treating febrile seizures in children: prospective randomized study. BMJ 2000; 321: 83-86.

3-Nordt SP, Clark RF. Midazolam: a review of therapeutic uses and toxicity. J Emerg Med 1997; 15(3): 357-365.

4-Hunter R. Status epilepticus. History, incidence and problems. Epilepsia 1959; 1:162-188.

5-Brown KK, Hussain IHN. Status epilepticus. I: Pathogenesis. Dev Med Child Neurol 1991; 33(1): 3-17.

6-Brown KK, Hussain IHN. Status epilepticus. II: Treatment. Dev Med Child Neurol 1991; 33: 97-109.

7-Treiman DM. The role of benzodiazepines in the management of status epilepticus. Neurology 1990; 40(5Suppl 2): 32-42.

8-Giang DW, McBride MC. Lorazepam versus diazepam for the treatment of status epilepticus. Pediatr Neurol 1988; 4(6): 358-361.

9-Leppik IE. Status epilepticus: The next decade. Neurology 1990; 40(5Sup $p / 2): 4-9$

10-Scheepers M, Scheepers B, Clarke M, Comish S, Ibitoye M. Is intranasal midazolam an effective rescue medication in adolescents and adults with severe epilepsy? Seizure 2000; 9: 417-421.

11-Holmes GL. Buccal route for benzodiazepines in treatment of seizures? Lancet 1999; 353: 608-609.

12-Chattopadhyay A, Morris B, Blackburn L, Wassmer E, Whitehouse W. Buccal midazolam and rectal diazepam for epilepsy. Lancet 1999; 353 1798.

13-Kanto JH. Midazolam: the first water-soluble benzodiazepine, pharmacology, pharmacokinetics and efficacy in insomnia and anesthesia. Pharmacolotherapy 1985; 5:138-155.

14-Baraff LJ . Conscious sedation of children. Ann Emerg Med 1994; 24: 1170 1172.

15-Hartgraves PM, Primosch RE. An effect of oral and nasal midazolam for pediatric dental sedation. J Dent Child 1994; 61: 175-181.

16-Taylor J W, Simon KB. Possible intramuscular midazolam-associated cardiorespiratory arrest and death. DICP 1990; 24: 695-697.

17-Wright SW, Chudnofsky CR, Dronen SC, et al. Comparison of midazolam and diazepam for conscious sedation in the emergency department. Ann Emerg Med 1993; 22: 201-205.

18-Doyle WL, Perrin L. Emergence delirium in a child given oral midazolam for conscious sedation. Ann Emerg Med 1994; 24: 1173-1175.

19-Feld LH, Negus JB, White PF. Oral midazolam preanesthetic medication in pediatric outpatients. Anesthiology 1990; 73: 831-834.

20-Ramoska Ea, Linkenheimer R, Glasgow C. Midazolam use in the emer gency department. J Emerg Med 1991; 9: 247-251.

21-Coughlin MW, Panuska HJ. Direct comparison of midazolam and diazepam for conscious sedation in outpatient oral surgery. Anesth Prog 1989; 36: $150-168$

22-Wyant M, Diamond BI, O'Neal E, Sloan A. The use of midazolam in acutely agitated psychiatric patients. Psychopharmacol Bull 1990; 26: 126-129.

23-Cole SG, Brozinsky S, Isenberg J L. Midazolam, a new more potent benzodiazepine, compared with diazepam: a randomized, doble-blind study of preendoscopic sedatives. Gastrointest Endosc 1983; 3: 219-222.

24-Knoester PD, J onker DM, Van Der Hoeven RT, Vermeij TA, Edelbroek PM, Brekelmans $G L$, et.al. Pharmacokinetics and pharmacodynamics of midazolam administered as a concentrated intranasal spray. A study in healthy volunteers. Br J Clin Pharmacol 2002; 53: 501-507.
25-Bjorkman S, Rigerman G, Idvall J. Pharmacokinetics of midazolam given as an intranasal spray to adult surgical patients. Br J Anaesth 1997; 79: 575-580.

26-Olivier J C, Djilani M, Fahmy S, Couet W. In situ nasal absorption of midazolam in rats. Int J Pharm 2001; 213: 187-192.

27-Karl HW, Rosenberger J L, Larach MG, Ruffle J M. Transmucosal administration of midazolam for premedication of pediatric patients. Comparison of the nasal and sublingual routes. Anesthesiology 1993; 78: 885-891.

28-Connors K, Terndrup TE. Nasal versus oral midazolam for sedation of anxious children undergoing laceration repair. Ann Emerg Med 1994; 24: 10751079 .

29-Anonymous. Midazolam-is antagonism justified? Lancet 1988; 2: 388-389.

30-Yaster M, Nichols DG, Deshpande J K, Wetzel RC. Midazolam fentanyl intravenous sedation in children: case report of respiratory arrest. Pediatrics 1990; 86: 463-467.

31-Bailey PL, Pace NL, Ashburn MA, Moll J WB, East KA, Stanley TH. Frequent hypoxemia and apnea after sedation with midazolam and fentanyl. Anesthesiology 1990; 73: 826-830.

32-Arcos GL. Midazolam-induced ventricular irritability. Anesthiology 1987; 67: 612 .

33-Yakel DL, Whittaker S, Elstad MR. Midazolam-induced angioedema and bronchoconstriction. Crit Care Med 1992; 20: 307-308.

34-Green CJ . Options for sedating children. West J Med 1993; 159: 596-597.

35-Lugo RA, Fishbein M, Nahata MC, Lininger B. Complication of intranasal midazolam. Pediatrics 1993; 92: 638.

36-Kendall J, Reynolds M, Goldberg R. Intranasal midazolam in patients with status epilepticus. ANN Emerg Med 1997; 29: 415-417.

37-Scott RC, Neville BGR, Besag FMC, Boyd SG. Nasal rather than rectal benzodiazepines en the management of acute childhood seizures. Dev Med Child Neurol 1997; 39: 137-138.

38-Scheepers M, Scheepers B, Clough P. Midazolam via the intranasal route: an effective rescue medication for severe epilepsy in adults with a learning disability. Seizure 1998; 7: 509-512.

39- Lahat E, Goldman M, Barr J, Eshel G, Berkovitch M. Intranasal midazolam with intravenous for childhood seizures. Lancet 1998; 352: 620

40-J eannet PY, Roulet E, Maeder-Ingvar M, Gehri M, J utzi A, Deonna T. Home and hospital treatment of acute seizures in children with nasal midazolam. Eur J Paediatr Neurol 1999; 3: 73-77.

41-Scott R, Besag F, Neville B. Buccal midazolam and rectal diazepam for treatment of prolonged seizures in childhood and adolescence: a randomized trial. Lancet 1999; 353: 623-626.

42-Fisgin T, Gürer Y, Tezic T, Zorlu P, Okuyaz C, Akgün D. Nasal midazolam effects on childhood acute seizures. J Child Neurol 2000; 15: 833-835.

43-Fisgin T, Gürer $Y$, Tezic T, Senbil N, Zorlu P, Okuyaz C, et al. Effects of intranasal midazolam and rectal diazepam on acute convulsions in children: prospective randomized study. J Child Neurol 2002; 17: 123-126.

44-Armijo J A, Herranz J L, Pena-Pardo MA, Adin J. Midazolam intranasal y bucal en el tratamiento de las convulsions agudas. Rev Neurol 2004; 38(5): 458-468.

45-Wallace SJ . Nasal benzodiazepines for management of acute childhood seizures? Lancet 1997; 349(25): 222.

46-Coughlin MW, Panuska HJ. Direct comparison of midazolam and diazepam for conscious sedation in outpatient oral surgery. Anesth Prog 1989; 36: 150-168

47-Canadian Task Force on the Periodic Health Examination: The periodic health examination. CMAJ 1979; 121: 1193-1254.

48-Sackett DL. Rules of evidence and clinical recommendations on use of antithrombotic agents. Chest 1986; 89(2): 2S-3S 